\title{
Intracranial haemorrhage in pregnancy
}

\author{
Jacob M Fairhall BSc (Med) MBBS* and Marcus A Stoodley PhD FRACS*† \\ ${ }^{*}$ Prince of Wales Medical Research Institute, University of NSW; ${ }^{\dagger}$ Australian School of Advanced Medicine, Macquarie University, Sydney, Australia
}

\begin{abstract}
Summary: Intracranial haemorrhage $(\mathrm{ICH})$ is a rare, yet potentially devastating event in pregnancy. There is a risk of maternal mortality or morbidity and a significant risk to the unborn child. The risk of haemorrhage increases during the third trimester and is greatest during parturition and the puerperium. ICH can be extradural, subdural, subarachnoid or intraparenchymal. Causes of bleeding include trauma, arteriovenous malformations, aneurysms, preeclampsia/eclampsia and venous thrombosis. Urgent neurosurgical conditions generally outweigh obstetric considerations in management decisions, although anaesthetic and surgical modifications can be made to minimize adverse effects to the fetus.
\end{abstract}

Keywords: stroke, intracerebral haemorrhage, pregnancy, cerebral aneurysms, subarachnoid haemorrhage, arteriovenous malformations

\section{INTRODUCTION}

Intracranial haemorrhage $(\mathrm{ICH})$ is a rare, yet potentially devastating event in pregnancy. There may be maternal mortality or significant morbidity; the risk to the unborn child is also significant. Urgent neurosurgical conditions usually outweigh obstetric considerations and the treatment of $\mathrm{ICH}$ is generally similar in pregnant and non-pregnant women. Effective decision-making demands a thorough knowledge of the physiology of pregnancy and the pathophysiology of certain obstetric conditions.

\section{EPIDEMIOLOGY}

A precise epidemiological perspective on $\mathrm{ICH}$ in pregnancy is difficult to obtain. Stroke complicates from nine to 26 per 100,000 pregnancies and deliveries. Pregnancy-related stroke encompasses both haemorrhagic and ischaemic aetiologies, with haemorrhage accounting for up to $38 \%$ of cases. ${ }^{1}$ In a retrospective examination of 50,700 deliveries, over a 17-year period, haemorrhage was identified as the cause of 13 of the 34 strokes identified. ${ }^{1}$ Aneurysmal subarachnoid haemorrhage (SAH) predominated as the main cause of $\mathrm{ICH}$; rupture of arteriovenous malformation (AVM) was the next most common source. The single intracerebral haemorrhage from disseminated intravascular coagulation in that study was immediately postpartum. In another retrospective study of 154 patients with $\mathrm{ICH}$ in pregnancy, $77 \%$ were aneurysmal and $23 \%$ were caused by AVMs. ${ }^{2}$ A review of pregnancy-related ICH in London in the 1950s and 1960s showed that of 52 haemorrhages, 21 were aneurysmal and 19 were related to AVMs. ${ }^{3}$ Less frequent

Correspondence to: Marcus A Stoodley, The Australian School of Advanced Medicine, Level 1, Dow Corning Building, 3 Innovation Road, Macquarie University, NSW 2109, Australia

Email: marcus.stoodley@asam.mq.edu.au causes of haemorrhage include preeclampsia/eclampsia, coagulopathy, trauma, and cerebral venous thrombosis.

Although ICH in pregnancy is a rare event, it remains a significant cause of maternal mortality. A large, retrospective study of births in Minnesota found no maternal ICH over a 24-year period encompassing 26,099 live births. ${ }^{4}$ However, other studies have reported a more significant haemorrhage risk. Barno et al. ${ }^{5}$ showed that haemorrhage related to AVMs and aneurysms accounted for $4.4 \%$ of all maternal deaths in a 23-year period, ranking as the third most common cause of deaths unrelated to obstetric causes. Of the maternal deaths from haemorrhage in this study, 51\% were attributed to SAH. A more recent study suggested that $\mathrm{ICH}$ may be responsible for up to $7.1 \%$ of all maternal mortality. ${ }^{6}$

The data are conflicting as to whether the risk of $\mathrm{ICH}$ is increased throughout pregnancy, but it seems likely that the rate of $\mathrm{ICH}$ increases during the third trimester. ${ }^{6-8}$ Bateman $^{6}$ reported a rate of pregnancy-related ICH of 7.1 per 100,000 at-risk-person-years compared with an equivalent nonpregnant rate of 5.0 per 100,000 at-risk-person-years. The risk of $\mathrm{ICH}$ appears to be greatest during parturition and in the puerperium. Kittner's population-based study suggested a relative risk of $\mathrm{ICH}$ of 2.5 during pregnancy and 28.3 in the puerperium. ${ }^{9}$

\section{VASCULAR PHYSIOLOGY IN PREGNANCY}

It is well appreciated that pregnancy and labour are hyperdynamic states. Blood volume increases in early pregnancy and reaches a plateau at 32 weeks, by which time blood volume has increased by $50 \%$. Plasma volume increases disproportionately during this time leading to a reduction in haematocrit. Although cardiac output increases by $30-50 \%$ over the first 24 weeks of pregnancy, systemic vascular tone falls and blood pressure decreases. During the first stage of labour, cardiac output increases by $50 \%$ from the pregnancy baseline. ${ }^{10}$ The 
mean arterial pressure also increases by up to $20 \%$ during uterine contractions. By $24 \mathrm{~h}$ after delivery, all haemodynamic variables are returned to the prelabour baseline. ${ }^{10}$

Normally, the blood brain barrier (BBB) prevents extravasation of proteins, sodium and potassium into the extracellular space of the brain. This uniquely alters local Starling's forces so that the osmotic pressure exerted by ions retained in the vascular compartment opposes transit of water into the brain. This feature prevents vasogenic oedema under usual conditions. ${ }^{11}$ Several studies have shown that pregnancy increases BBB permeability in the presence of hypertension, with some regional variation. $^{12}$

Cerebral blood flow (CBF) and perfusion pressure have been measured indirectly in pregnancy with transcranial Doppler middle cerebral artery (MCA) flow measurements and velocity-encoded magnetic resonance imaging (MRI). As gestation progresses, MCA flow decreases and CBF decreases. ${ }^{13}$ Some authors have found that CBF decreases by $20 \%$ in the latter stages of pregnancy and posit that vascular resistance falls to minimize the change in perfusion. ${ }^{14}$ It is possible that the range of cerebral autoregulation changes during pregnancy and the relative hypertension of preeclampsia and eclampsia leads to rupture of vessels at a lower pressure than in the non-pregnant women.

Pregnancy is also a state where changes occur in the haemostatic system. Typically, there is hypercoagulability with alterations in both mechanisms of coagulation and fibrinolysis. Procoagulant factors including factors VII, VIII and X, von Willebrand's factor and fibrinogen are increased. Protein S decreases and fibrinolytic activity is also decreased. ${ }^{15}$ In the puerperium, physiological intravascular volume changes in the context of this haemostatic milieu may predispose further to thrombotic events.

\section{TYPES OF ICH}

ICH may be classified anatomically as extradural, subdural, subarachnoid or intracerebral. Extradural and subdural haemorrhages are usually related to trauma, as are some intracerebral haemorrhages. Spontaneous SAH is usually caused by aneurysmal rupture. Spontaneous intracerebral haemorrhage in obstetric patients is most often caused by AVM, hypertension or venous occlusion.

\section{Extradural haemorrhage}

The chief cause of extradural haemorrhage is trauma. This type of haemorrhage is commonly associated with skull fracture and injury to the middle meningeal artery. Primary brain damage is usually minimal and the prognosis is good with timely surgical treatment. Presentation is usually precipitous with rapidly deteriorating conscious state and urgent operative treatment is mandatory.

\section{Subdural haemorrhage}

Acute subdural haemorrhage (SDH) is usually a result of severe traumatic brain injury and carries a poor prognosis. The epidemiology and treatment of this injury in pregnancy are no different from the non-pregnant population. Chronic SDH is extremely uncommon in pregnancy, as it is a disease usually affecting the elderly. There are several reports in the literature referring to SDH after epidural or spinal anaesthesia, ${ }^{16,17}$ presumably related to intracranial cerebrospinal fluid hypovolaemia and low intracranial pressure causing stretching and tearing of bridging veins between the cortex and dural venous sinuses. Surgery for chronic SDH can be carried out under local anaesthesia, which would be preferable to general anaesthesia in a pregnant patient. Aneurysm rupture may present as a spontaneous acute $\mathrm{SDH}$, although this is uncommon.

\section{Subarachnoid haemorrhage}

\section{Epidemiology and clinical features}

The first reported case of SAH in pregnancy was in $1899 .{ }^{18}$ Since this time, much of the literature related to its occurrence and treatment in pregnancy has been anecdotal or small series. The overall frequency of pregnancy-related $\mathrm{SAH}$ during pregnancy is thought to be $0.01-0.03 \% .{ }^{19}$ Up to $90 \%$ of these SAHs occur during pregnancy, $2 \%$ during delivery and $8 \%$ during the puerperium. ${ }^{2}$ There appears to be a tendency for a higher frequency of SAH with advancing gestation, suggesting that haemodynamic or other physiological changes in pregnancy do influence the rate of aneurysmal growth or rupture.

Pregnancy does not seem to alter the clinical presentation of $\mathrm{SAH}$. One-third of patients present in a poor neurological grade with coma or depressed consciousness. Those patients who present with a preserved level of consciousness typically describe sudden onset of severe headache invariably associated with vomiting. Focal neurological deficits may be present, especially if there is associated intracerebral haemorrhage. The initial symptoms may be similar to eclampsia or preeclampsia. $^{20}$

Immediate computed tomography $(\mathrm{CT})$ is the investigation of choice for diagnosing SAH. There may be a temptation to use MRI rather than CT in pregnant patients, but MRI is not sensitive in the detection of acute subarachnoid blood. However, if the presentation is delayed, the sensitivity of CT is reduced and the changes in haemoglobin and red cell breakdown make MRI a more sensitive method of detecting the presence of subarachnoid blood. If the diagnosis remains in doubt after CT and/or MRI, a lumbar puncture with spectrophotometric examination of cerebrospinal fluid for bilirubin will provide definitive evidence of the presence or absence of subarachnoid haemorrhage. Once a diagnosis of SAH has been made, further investigation is directed at determining the underlying cause of the haemorrhage. Diagnosis of an aneurysm and assessment of aneurysm morphology is performed with digital subtraction angiography (DSA) or, increasingly, CT-angiography. These investigations should be performed with appropriate shielding of the fetus from ionizing radiation.

The natural history of untreated aneurysmal $\mathrm{SAH}$ is dire, with a $65 \%$ mortality rate at one year. The risk of rebleeding from a ruptured aneurysm during pregnancy is up to $50 \%$, with the greatest risk in the initial period after haemorrhage. Expeditious treatment of the ruptured aneurysm is recommended in all but the most moribund patients.

\section{Treatment}

A main goal of treatment in aneurysmal SAH is the prevention of repeat haemorrhage. Aneurysm occlusion can be achieved by 
endovascular or open surgical techniques. There has been a shift over the last 10 years to endovascular treatment of ruptured aneurysms. Initial concerns were raised about radiation exposure in pregnant patients; however, these have been largely overcome. ${ }^{21}$ The long-term durability of endovascular aneurysm repair in pregnant patients is unclear. The physiological states that may influence aneurysm change or rupture in pregnancy are insufficiently understood to extrapolate the coiling data from non-pregnant patients to pregnant patients. It is currently reasonable, nonetheless, to make similar treatment decisions for pregnant women as for age-matched nonpregnant women.

Bed rest and a quiet environment should be maintained before the aneurysm has been secured. Normovolaemia should be maintained and consideration is given to calcium channel blockade. Delayed ischaemic deficits due to arterial spasm remain a cause of disability after SAH. It has been suggested that pregnancy itself may be protective for vasospasm owing to rheological changes, ${ }^{22}$ although this is contentious.

\section{Intracerebral haemorrhage}

\section{Arteriovenous malformation}

Epidemiology and clinical features

AVMs are the chief cause of intracerebral haemorrhage in pregnancy. ${ }^{7}$ The community prevalence of AVMs is $15-18$ per 100,000 and it could be assumed that the same prevalence applies to pregnant women. ${ }^{23}$ The risk of haemorrhage from a previously unruptured AVM in pregnant women is estimated at $3.5 \%$ per annum against $3.1 \%$ per annum in non-pregnant women of child-bearing age, suggesting that pregnancy is not a significant risk factor for haemorrhage. If, however, there is a haemorrhage from an AVM during pregnancy, the risk of further haemorrhage is $27 \%$, which is higher than the rebleeding rate in non-pregnant patients. ${ }^{24}$

\section{Management}

The appropriate management of a ruptured AVM in pregnancy is contentious. Dias et l. $^{2}$ reported an overall maternal mortality rate of $28 \%$ after AVM haemorrhage, yet demonstrated no significant difference between women who had their lesion surgically treated during pregnancy and those who did not. Coupled with the uncertainty surrounding these lesions' natural history is the lack of data pertaining to AVM surgery in pregnant patients. In non-pregnant patients, blood pressure is normally kept low during surgery and in the postoperative period to avoid breakthrough haemorrhage from chronically dilated vessels. In a pregnant patient, this would need to be balanced against the need to maintain placental perfusion. It is sensible to expedite delivery by caesarean section where AVM rupture occurs at an advanced gestational stage the AVM can then be treated along conventional lines. It is probably appropriate to deliver by caesarean section cases where an AVM has ruptured but not been treated, in order to avoid Valsalva manoeuvres and transient blood pressure surges that may lead to further haemorrhage, although the relative risk of this is unquantifiable. Vaginal delivery is not precluded after an AVM has been resected, although fragile vessels in the brain at the resection margin may remain at higher risk of haemorrhage in the first few weeks after an AVM has been removed.

\section{Preeclampsia and eclampsia}

Preeclampsia classically presents with hypertension of greater than $140 / 90 \mathrm{mmHg}$ and proteinuria $>300 \mathrm{mg} /$ day. Preeclampsia is often clinically silent, but may cause symptoms of neurological dysfunction such as headache, visual disturbance, and impairment of consciousness. Eclampsia is the occurrence of seizures in the context of preeclampsia and is often, but not invariably, preceded by the above neurological symptoms. Some patients also have stroke-like episodes of focal neurological deficit. Systemic vascular resistance increases in preeclampsia with a resultant hypertension and reduction in cardiac output and plasma volume. ${ }^{2,26}$ Major cerebrovascular changes in eclampsia are thought to be similar to those of hypertensive encephalopathy with oedema and a state of cerebral hyperperfusion. ${ }^{13}$ Generally the imaging features are reversible with neuroimaging characteristics of cerebral oedema ${ }^{27}$ and a predilection for the posterior parietal and occipital lobes. ${ }^{13}$

Intracerebral haemorrhage has been found in up to $40 \%$ of patients with eclampsia in autopsy series. ${ }^{28}$ Eclampsia is an important cause of serious haemorrhagic stroke - in an intensive care admission study, four of 11 pregnant patients admitted with haemorrhagic stroke were eclamptic. ${ }^{29}$ Haemorrhages may be extensive or petechial. It is thought that haemorrhage is probably a result of severe hypertension in conjunction with increased cerebrovascular resistance and loss of autoregulation. Eclampsia is best treated with Magnesium sulphate, control of hypertension, and delivery of the infant.

Management of the intracerebral haemorrhage follows standard neurosurgical principles. Surgical evacuation of a clot is indicated if there is a declining conscious state or worsening neurological deficit (Figure 1). A non-operative approach is

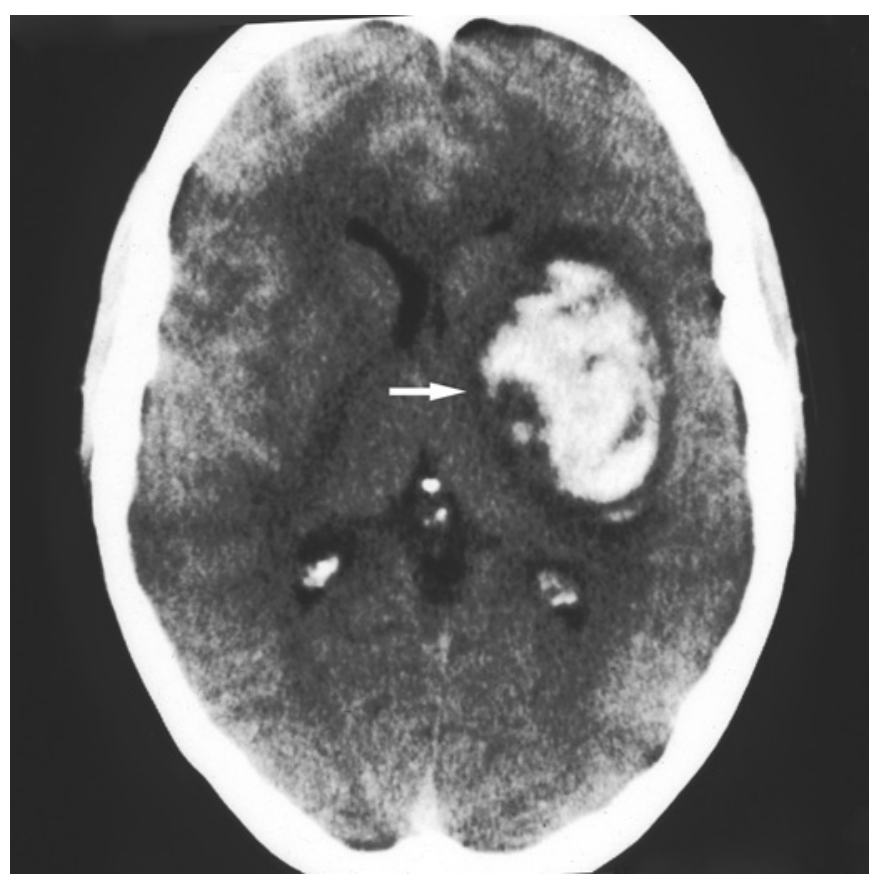

Figure 1 Non-contrast computed tomography demonstrating left putaminal haemorrhage (arrow). This 35-year-old patient was being treated for depression with a monoamine oxidase inhibitor (MAOI) during pregnancy. After delivery, she ate a large amount of cheese and had an MAOI hypertensive crisis. She had a depressed conscious state on admission and was treated with craniotomy and evacuation of the haematoma. She made a full neurological recovery 
appropriate in cases of devastating haemorrhage that is nonrecoverable, although surgery might be considered in an effort to prolong the life of the mother in the interests of the fetus. The role of surgery is less clear in other cases. A large trial comparing early surgery with medical management in primary intracerebral haemorrhage did not support the use of early surgery in this intermediate group. ${ }^{30}$ However, the trial should not be interpreted as demonstrating that surgery has no role: it only demonstrates that in cases where the role of surgery is unclear, there is no benefit from early surgical intervention. Of course, surgery remains the appropriate treatment when there is progression of the clinical state to one of deteriorating consciousness or progressive neurological deficit.

\section{Cerebral venous thrombosis}

Epidemiology and clinical features

Pregnancy and particularly the puerperium are significant risk factors for cerebral venous thrombosis. Pregnant patients with prothrombotic disorders such as protein C or S deficiency are at particular risk. Caesarean section, hypertension, dehydration, and infections also predispose to thrombosis. ${ }^{31}$

Venous sinus and cortical venous thrombosis result in increased venous pressure in the brain, cerebral oedema, and often venous hypertensive haemorrhage (Figure 2). Patients may present with headache of acute or subacute onset, seizures, decreased level of consciousness, or focal neurological deficits. As in all cases of sudden severe headache, CT is the imaging of first choice, which may show poorly defined low density that is not restricted to an arterial distribution. CT venography is an excellent tool to visualize the dural sinuses and can quickly confirm the diagnosis.

Cerebral venous sinus thrombosis can result in mortality rates of up to $36 \% .^{32,33}$ In a retrospective appraisal of obstetric patients with venous thrombosis, ${ }^{32} 67$ presented in the puerperium and only five during pregnancy. Up to $60 \%$ of pregnant patients with cerebral venous sinus thrombosis have some degree of intracerebral haemorrhage or haemorrhagic venous infarction. This suggests that haemorrhage may be more common in obstetric groups as large population-based studies have shown a haemorrhage rate of only $39 \% .^{34}$

\section{Treatment}

Generally, anti-coagulation is the main treatment of cerebral venous thrombosis whether or not there is co-existent intracerebral haemorrhage. Full anti-coagulation is generally safe from $24 \mathrm{~h}$ postpartum. Both low molecular weight heparin and warfarin are safe during breast-feeding. Surgical evacuation of large haemorrhages will occasionally be required. An important preventive measure is to ensure adequate hydration in the puerperium.

\section{Neoplasia}

Haemorrhagic metastases in pregnancy are extremely rare. Metastases from renal cell carcinoma or melanoma may present with intracerebral haemorrhage. Gestational choriocarcinoma is usually confined to molar pregnancy but may be a consideration in the puerperium following an otherwise normal delivery. ${ }^{35}$ Treatment should be held in abeyance if possible, but if mass effect is pronounced, resection of the lesion and haemorrhage is appropriate.

\section{Moyamoya disease}

Moyamoya is a rare condition characterized by spontaneous gradual occlusion of the arteries in the circle of Willis. Perforating arteries dilate in response to the chronic ischaemia and rupture of these enlarged fragile vessels is a cause of intracerebral haemorrhage in young adults. There are several case studies of ICH during pregnancy in patients with moyamoya disease. ${ }^{36,37}$ Intraventricular haemorrhage is common in the setting of moyamoya disease and is managed with ventricular drainage. Haematomas with significant mass effect should be removed, although the fragile vessels pose a risk of recurrent haemorrhage. Management of the chronic ischaemia is a complex problem, often requiring revascularization surgery with bypass grafts. Unless there are transient ischaemic attacks indicating a high-risk of imminent ischaemic stroke, revascularization surgery may best be delayed until after delivery.

\section{Cavernous malformations}

Cavernous malformations, or 'cavernomas,' are a form of vascular malformation comprised of thin-walled vascular channels. The blood flow in these lesions is much less than in AVMs and haemorrhages from them are usually of low volume. Presentation is often subacute with seizures or focal
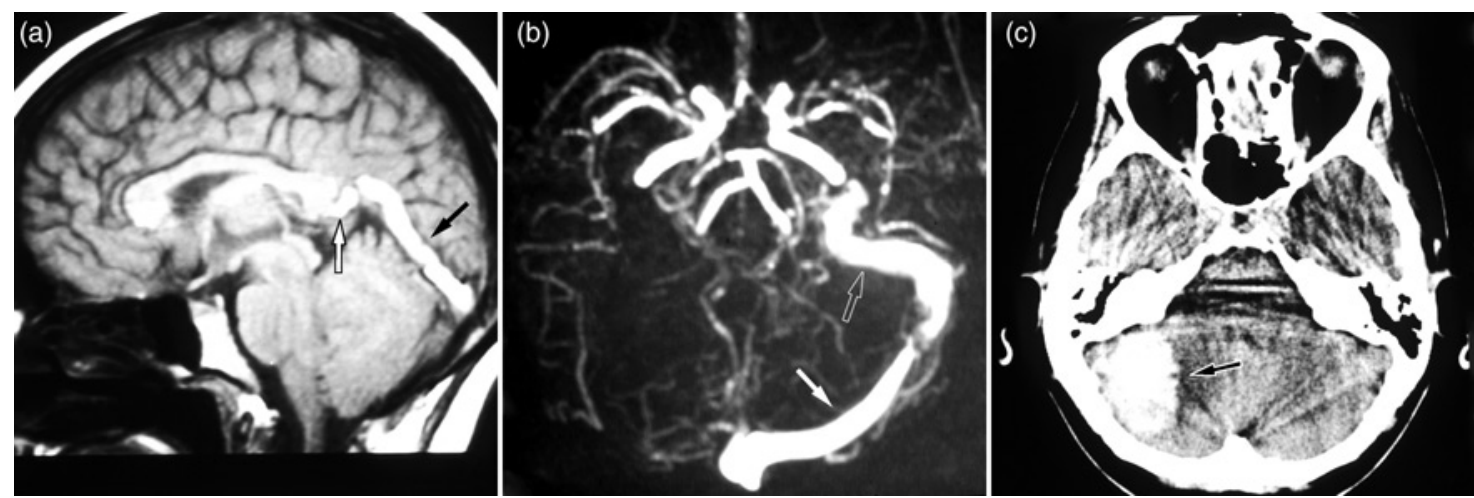

Figure 2 Dural venous sinus thrombosis resulting in intracranial haemorrhage. (a) T1-weighted non-contrast sagittal magnetic resonance imaging (MRI) demonstrating thrombosis of the vein of Galen (white arrow) and straight sinus (arrow). (b) MR venogram showing patent left transverse (arrow) and sigmoid (black arrow) sinuses and absent signal on the left, indicating occlusion. (c) Non-contrast computed tomography showing intraparenchymal haemorrhage in the right cerebellar hemisphere (arrow) 

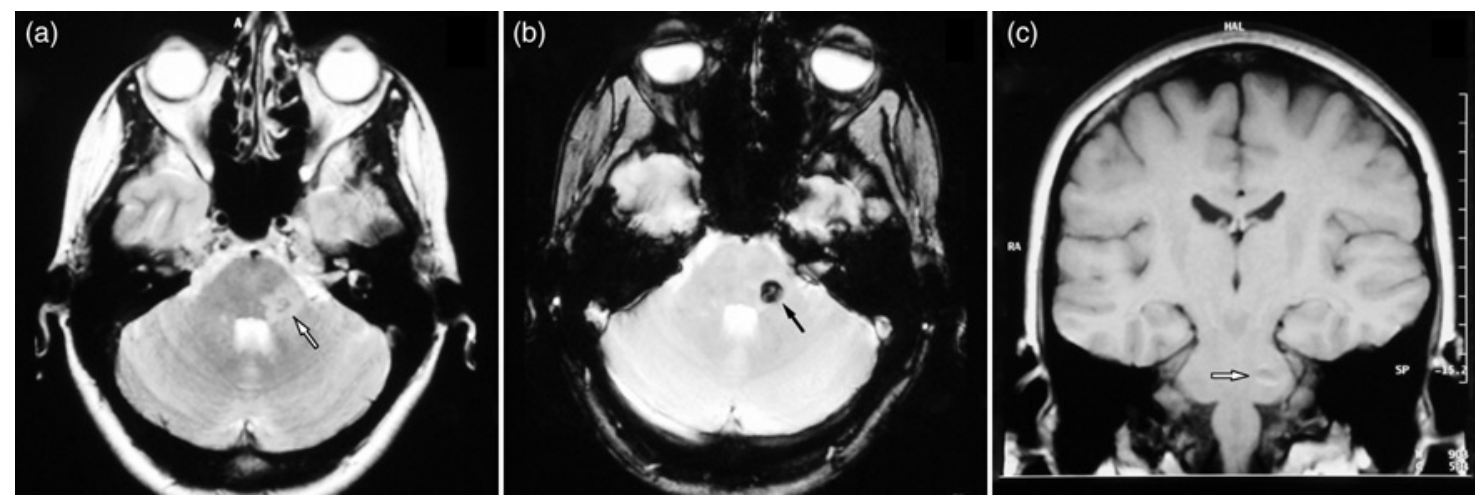

Figure 3 Brainstem cavernous malformation with oedema and small intra-axial haemorrhage. This woman of 21 weeks' gestation presented with headache and hemianaesthesia. (a) T2-weighted axial magnetic resonance imaging (MRI) demonstrating cavernoma in the right middle cerebellar peduncle with prominent perilesional oedema (arrow). (b) Gradient echo sequence axial MRI showing a cavernous malformation with haemorrhage and haemosiderin deposits (arrow). (c) T1-weighted coronal MRI with acute haemorrhage in the left middle cerebellar peduncle, surrounding a cavernous malformation (arrow)

neurological deficit (Figure 3). MRI is the best imaging method for these lesions, which are angiographically occult.

Some authors suggest that pregnancy is associated with an increased risk of haemorrhage from cavernous malformations and also with an increase in their size. ${ }^{38}$ However, there are fewer than 30 reports in the literature and it is difficult to draw any firm conclusions. ${ }^{39-42}$ In a single unit study, seven of 62 women treated for brainstem cavernoma were pregnant. ${ }^{41}$ These women averaged 2.4 haemorrhages before surgical treatment; only one patient required surgery while pregnant.

Urgent surgical removal is not typically required and any treatment is ideally deferred until after delivery.

\section{INVESTIGATIONS}

\section{Computed tomography}

CT is the mainstay for urgent investigation of suspected intracranial pathology, especially if haemorrhage is suspected. It is a quick study, and particularly sensitive for ICH. It is always prudent to limit the amount of ionizing radiation received during pregnancy; however, it is estimated that a noncontrast head CT results in shielded fetal radiation exposure of less than $0.005 \mathrm{mGy}$. This is far less than the $100 \mathrm{mGy}$ generally considered the threshold for the development of fetal malformations from radiation exposure. ${ }^{43}$ The incidence of adverse fetal effect with contrast media appears low, although there is a small risk of neonatal hypothyroidism as iodinebased contrast media may cross the placenta. CT is the preferred investigation for detection of acute $\mathrm{ICH}$; it is also superior to MRI for non-invasive arteriography and venography.

\section{Magnetic resonance maging}

MRI has been used in fetal diagnosis for over 25 years and its safety is well established. ${ }^{44}$ MRI also has a greater sensitivity than CT in demonstrating non-acute haemorrhage. Given the fact that there is no ionizing radiation, this modality may be superior to CT when repeated surveillance of a previously detected $\mathrm{ICH}$ is required.

\section{Digital subtraction angiography}

DSA is a sensitive and specific test for cerebral aneurysms; however, radiation doses from DSA are appreciably higher than from CT. Feygelman estimates up to $3.6 \mathrm{mSv}$ cranial exposure with angiography, a value 5-8 fold higher than that for $\mathrm{CT} ;{ }^{45}$ it follows that fetal exposure would still be less than 0.025 mGy. $^{46}$

Thus ionizing radiation exposure to the fetus is minimal in the investigation of $\mathrm{ICH}$ and its endovascular treatment, if applicable.

\section{MANAGEMENT}

\section{General principles of management}

The management principles for ICH are similar in pregnant and non-pregnant patients. After resuscitation, the first priority is the evacuation of any haemorrhage causing critical mass effect; the next priority is to investigate and address the underlying source of the haemorrhage such as aneurysm or AVM. Attention then turns to preventing further haemorrhage. It is important that management of these patients takes place in facilities where there is appropriate neurosurgical, obstetric, neurological and neuroradiological expertise. In the event that surgery is undertaken, precautions related to the pregnancy should be followed.

In some centres it might be considered appropriate to manage certain patients in a stroke unit, although generally the management of acute haemorrhage is reserved for the neurosurgical and intensive care units. The multidisciplinary care provided in a stroke unit does produce better outcomes for the rehabilitation phase after stroke, so where possible patients should be managed in such units after the acute phase has resolved. $^{47}$

\section{Operative considerations}

Inferior vena cava compression in the supine position may be significant after 24 weeks pregnancy. During surgery, the woman's trunk should therefore be positioned in a partial left lateral position where possible. 
Hypothermia as a neuroprotective strategy has been a traditional neuroanaesthetic adjunct in some centres when treating aneurysms and AVMs. This generally serves to decrease CBF and metabolic requirements. Moderate hypothermia (30$32{ }^{\circ} \mathrm{C}$ ) reduces fetal heart rate by up to $25 \%$. There is concern that placental oxygen transfer is less efficient at low temperatures and uterine contractions may be provoked. In a retrospective review of cardiopulmonary bypass in pregnancy, fetal mortality was substantially increased when hypothermia was used. $^{48}$ Recent evidence suggests that any benefit from hypothermia for aneurysm surgery is slender, if present at all; ${ }^{49}$ thus, it is recommended that hypothermia be avoided for all neurosurgical procedures in pregnancy.

Hypotension is sometimes used during the surgical approach to aneurysms in order to reduce the risk of intra-operative rupture. It is also commonly used in AVM surgery. Sodium nitroprusside has been associated with high fetal cyanide levels; hypotension also has a deleterious effect on uterine and placental perfusion and may lead to fetal anoxia. It is preferable to use temporary proximal clipping as an alternative method of softening the aneurysm as this does not affect the placental and fetal circulations.

Diuretic agents such as mannitol or frusemide are generally used to reduce intracranial tension and improve operative conditions. However, their use may result in significant negative fluid shifts affecting both mother and fetus, which may result in hypotension and placental hypoperfusion.

Neostigmine may be used for the reversal of muscle relaxants in neurosurgery. Glycopyrrolate or atropine is often given with neostigmine in order to block its muscarinic effects; glycopyrrolate does not cross the placenta whereas neostigmine does. Profound fetal bradycardia has occurred where glycopyrrolate has been given instead of atropine. Atropine should therefore be used in preference to glycopyrrolate when using neostigmine. ${ }^{50}$

Hyperventilation is often used during neurosurgery to produce hypocarbia, which causes vasoconstriction, reduction of intracranial blood volume, and reduced intracranial pressure. In pregnant patients, this may lead to decreased placental oxygen transfer; however, this should not be significant with mild hyperventilation (partial pressure $\mathrm{CO}_{2}$ of $30-35 \mathrm{mmHg}$ ). Profound hypocarbia should be avoided.

\section{Pharmacology}

A number of medications may be routinely administered to patients undergoing urgent surgery for ICH. These may include anticonvulsants, calcium channel antagonists or osmotic diuretics. Steroids are not routinely used in the management of ICH.

\section{Anticonvulsants}

Phenytoin is commonly administered to neurosurgical patients yet its deleterious effects in pregnancy, the fetal hydantoin syndrome, are well described. ${ }^{51}$ The cluster of abnormalities includes microcephaly, craniofacial abnormalities, distal phalangeal hypoplasia and developmental delay. Similar abnormalities have been seen with barbiturate use. Neural tube defects have been observed with sodium valproate. Carbamazepine may have the least risk of teratogenicity, but it is not commonly used in acute neurosurgical practice because of the lack of an intravenous preparation.

\section{Calcium channel antagonists}

Nimodipine is used commonly after aneurysmal SAH. It has been shown in some randomized trials to improve outcome in this situation. A Cochrane review showed that calcium channel antagonists provide only a 5.1\% reduction in risk of poor outcome. Nimodipine has been shown to be teratogenic in animals, yet a prospective, multicentre study of exposure of calcium channel antagonists in the first trimester of pregnancy showed no increase in the risk of major congenital malformations, ${ }^{52}$ and nifedipine and amlodipine are commonly used in pregnancy to treat hypertension without any evidence to suggest detrimental fetal effects. Although it is recommended by some to give nimodipine routinely for all expectant women with $\mathrm{SAH}$, it would be nonetheless prudent to give nimodipine only to those patients with a large volume of subarachnoid blood, who are at high risk of vasospasm. In these patients, careful haemodynamic monitoring is necessary to ensure that hypotension does not jeopardize placental perfusion.

\section{ETHICAL ASPECTS OF MATERNAL IMPAIRMENT AFTER ICH}

When brain death (BD) or persistent vegetative state (PVS) occur in pregnancy, they pose significant ethical, medical and legal challenges. There are nearly 30 reports in the literature of pregnancy being supported until parturition in the event of severe maternal impairment (BD or PVS).$^{53-55}$ Of 10 reported brain deaths in pregnancy, six were caused by ICH of an unspecified type. One was caused by traumatic brain injury that may have had associated haemorrhage. In the 17 reports of pregnancy-associated PVS, three were related to AVM rupture and eight related to traumatic brain injury. Fetal outlook has been generally good in the PVS group, with at least 10 of the 17 reported infants achieving normal outcomes; one infant died, two were developmentally delayed and four infants had no outcome reported. ${ }^{53}$ The fetal outcome has been similar in the reports of brain death associated with pregnancy with all infants surviving. It is possible that a reporting bias exists to account for the optimistic literature in these cases.

In the context of brain death, the longest period of maternal support was 107 days. ${ }^{56}$ Although it is considered generally preferable to attain a gestational age of at least 32 weeks before considering delivery in this circumstance, viable infants can be delivered at 24 weeks. This means that prolongation of support could be considered for cases of maternal brain death where gestational age is greater than 10 weeks. Of course, the physiological challenges to maternal support mean that the likelihood of successful delivery is much higher the closer to term brain death occurs. ${ }^{57}$

After declaration of $\mathrm{BD}$, the rights of the mother as an autonomous legal entity are extinguished but the rights of the fetus are not well established. The principles guiding care should be the premorbid wishes of the mother weighed against the likelihood of successful outcome and the wishes of surviving family. It should be considered that maternal support in this circumstance is experimental, and ethical and legal advice should be sought.

\section{CONCLUSION}

$\mathrm{ICH}$, although rare, is an important cause of morbidity and mortality in pregnancy. Treatment decisions should be based 
on the same principles as those for non-pregnant patients with awareness of the influence that pregnancy has on certain conditions.

\section{REFERENCES}

1 Jaigobin C, Silver FL. Stroke and pregnancy. Stroke 2000;31:2948-51

2 Dias MS, Sekhar LN. Intracranial hemorrhage from aneurysms and arteriovenous malformations during pregnancy and the puerperium. Neurosurgery 1990;27:855-65; discussion 65-6

3 Amias AG. Cerebral vascular disease in pregnancy. I. Haemorrhage. J Obstet Gynaecol Br Commonwealth 1970;77:100-20

4 Wiebers DO, Whisnant JP. The incidence of stroke among pregnant women in Rochester, Minn, 1955 through 1979. JAMA 1985;254:3055-7

5 Barno A, Freeman DW. Maternal deaths due to spontaneous subarachnoid hemorrhage. Am J Obstet Gynecol 1976;125:384-92

6 Bateman BT, Schumacher HC, Bushnell CD, et al. Intracerebral hemorrhage in pregnancy: frequency, risk factors, and outcome. Neurology 2006;67:424-9

7 Liang CC, Chang SD, Lai SL, Hsieh CC, Chueh HY, Lee TH. Stroke complicating pregnancy and the puerperium. Eur J Neurol 2006;13:1256-60

8 Davie CA, O'Brien P. Stroke and pregnancy. J Neurol Neurosurg Psychiatry 2008;79:240-5

9 Kittner SJ, Stern BJ, Feeser BR, et al. Pregnancy and the risk of stroke. N Engl J Med 1996;335:768-74

10 Robson SC, Dunlop W, Boys RJ, Hunter S. Cardiac output during labour. $\mathrm{Br}$ Med J (Clin Res Edn) 1987;295:1169-72

11 Kimelberg HK. Water homeostasis in the brain: basic concepts. Neuroscience 2004;129:851-60

12 Euser AG, Cipolla MJ. Cerebral blood flow autoregulation and edema formation during pregnancy in anesthetized rats. Hypertension 2007;49:334-40

13 Cipolla MJ. Cerebrovascular function in pregnancy and eclampsia. Hypertension 2007;50:14-24

14 Zeeman GG, Hatab M, Twickler DM. Maternal cerebral blood flow changes in pregnancy. Am J Obstet Gynecol 2003;189:968-72

15 Brenner B. Haemostatic changes in pregnancy. Thromb Res 2004;114:409-14

16 Mashour GA, Schwamm LH, Leffert L. Intracranial subdural hematomas and cerebral herniation after labor epidural with no evidence of dural puncture. Anesthesiology 2006;104:610-2

17 Zeidan A, Farhat O, Maaliki H, Baraka A. Does postdural puncture headache left untreated lead to subdural hematoma? Case report and review of the literature. Int J Obstet Anesthesia 2006;15:50-8

18 Lazard EM. A case of cerebral hemorrhage following labor. Philadelphia Med J 1899;4:1091-2

19 Stoodley MA, Weir BKA. Pregnancy and Aneurysms. In: Management of Cerebral Aneurysms. 2003, pp. 347-55. WB Saunders, Orlando

20 Stoodley MA, Macdonald RL, Weir BK. Pregnancy and intracranial aneurysms. Neurosurg Clin N Am 1998;9:549-56

21 Marshman LA, Aspoas AR, Rai MS, Chawda SJ. The implications of ISAT and ISUIA for the management of cerebral aneurysms during pregnancy. Neurosurg Rev 2007;30:177-80; discussion 80

22 Giannotta SL, Daniels J, Golde SH, Zelman V, Bayat A. Ruptured intracranial aneurysms during pregnancy. A report of four cases. J Reprod Med 1986;31:139-47

23 Al-Shahi R, Fang JSY, Lewis SC, Warlow CP. Prevalence of adults with brain arteriovenous malformations: a community based study in Scotland using capture-recapture analysis. J Neurol Neurosurg Psychiatry 2002;73:547-51

24 Sadasivan B, Malik GM, Lee C, Ausman JI. Vascular malformations and pregnancy. Surg Neurol 1990;33:305-13

25 Ganzevoort W, Rep A, Bonsel GJ, de Vries JI, Wolf H. Plasma volume and blood pressure regulation in hypertensive pregnancy. J Hypertens 2004;22:1235-42

26 Walker JJ. Pre-eclampsia. Lancet 2000;356:1260-5

27 Mas JL, Lamy C. Stroke in pregnancy and the puerperium. J Neurol 1998;245:305-13

28 Richards A, Graham D, Bullock R. Clinicopathological study of neurological complications due to hypertensive disorders of pregnancy. J Neurol Neurosurg Psychiatry 1988;51:416-21

29 Skidmore FM, Williams LS, Fradkin KD, Alonso RJ, Biller J. Presentation, etiology, and outcome of stroke in pregnancy and puerperium. J Stroke Cerebrovasc Dis 2001;10:1-10

30 Mendelow AD, Gregson BA, Fernandes HM, et al. Early surgery versus initial conservative treatment in patients with spontaneous supratentorial intracerebral haematomas in the International Surgical Trial in Intracerebral Haemorrhage (STICH): a randomised trial. Lancet 2005; 365:387-97

31 Lanska DJ, Kryscio RJ. Risk factors for peripartum and postpartum stroke and intracranial venous thrombosis. Stroke 2000;31:1274-82

32 Cantú C, Barinagarrementeria F. Cerebral venous thrombosis associated with pregnancy and puerperium. Review of 67 cases. Stroke 1993;24: 1880-4

33 Canhao P, Ferro JM, Lindgren AG, Bousser MG, Stam J, Barinagarrementeria F. Causes and predictors of death in cerebral venous thrombosis. Stroke 2005;36:1720-5

34 Girot M, Ferro JM, Canhao P, et al. Predictors of outcome in patients with cerebral venous thrombosis and intracerebral hemorrhage. Stroke 2007;38:337-42

35 Huang CY, Chen CA, Hsieh CY, Cheng WF. Intracerebral hemorrhage as initial presentation of gestational choriocarcinoma: a case report and literature review. Int J Gynecol Cancer 2007;17:1166-71

36 Enomoto H, Goto H. Moyamoya disease presenting as intracerebral hemorrhage during pregnancy: case report and review of the literature. Neurosurgery 1987;20:33-5

37 Karasawa J, Kikuchi H, Furuse S. Subependymal hematoma in "Moyamoya" disease. Surg Neurol 1980;13:118-20

38 Safavi-Abbasi S, Feiz-Erfan I, Spetzler RF, et al. Hemorrhage of cavernous malformations during pregnancy and in the peripartum period: causal or coincidence? Case report and review of the literature. Neurosurg 2006;21:e12

39 Pozzati E, Musiani M. Cavernous hemangioma. J Neurosurg 1998;89:498-9

40 Flemming KD, Goodman BP, Meyer FB. Successful brainstem cavernous malformation resection after repeated hemorrhages during pregnancy. Surg Neurol 2003;60:545-7; discussion 7-8

41 Porter RW, Detwiler PW, Spetzler RF, et al. Cavernous malformations of the brainstem: experience with 100 patients. J Neurosurg 1999;90:50-8

42 Robinson JR, Awad IA, Little JR. Natural history of the cavernous angioma. J Neurosurg 1991;75:709-14

43 ICRP. Pregnancy and medical radiation. Ann ICRP 2000;30:ii-viii, 1-43

44 Smith FW, Adam AH, Phillips WD. NMR imaging in pregnancy. Lancet 1983;1:61-2

45 Feygelman VM, Huda W, Peters KR. Effective dose equivalents to patients undergoing cerebral angiography. AJNR Am J Neuroradiol 1992;13:845-9

46 Murakami M, Nonaka N, Hirata Y, et al. Ruptured arteriovenous malformation during pregnancy with special reference to diagnostic X-ray exposure. Case report. Neurol Med Chir (Tokyo) 1990;30(11 Spec No):913-7

47 Stroke Unit Trialists' Collaboration. Organised inpatient (stroke unit) care for stroke. Cochrane Database of Systematic Reviews 2006, Issue 4

48 Pomini F, Mercogliano D, Cavalletti C, Caruso A, Pomini P. Cardiopulmonary bypass in pregnancy. Ann Thorac Surg 1996;61:259-68

49 Todd MM, Hindman BJ, Clarke WR, Torner JC, Investigators et al. Mild intraoperative hypothermia during surgery for intracranial aneurysm. $N$ Engl J Med 2005;352:135-45

50 Kuczkowski KM. Nonobstetric surgery during pregnancy: what are the risks of anesthesia? Obstet Gynecol Surv 2004;59:52-6

51 Hanson JW, Smith DW. The fetal hydantoin syndrome. J Pediatr 1975;87:285-90

52 Magee LA, Schick B, Donnenfeld AE, et al. The safety of calcium channel blockers in human pregnancy: a prospective, multicenter cohort study. Am J Obstet Gynecol 1996;174:823-8

53 Bush MC, Nagy S, Berkowitz RL, Gaddipati S. Pregnancy in a persistent vegetative state: case report, comparison to brain death, and review of the literature. Obstet Gynecol Surv 2003;58:738-48

54 Chiossi G, Novic K, Celebrezze JU, Thomas RL. Successful neonatal outcome in 2 cases of maternal persistent vegetative state treated in a labor and delivery suite. Am J Obstet Gynecol 2006;195:316-22

55 Powner DJ, Bernstein IM. Extended somatic support for pregnant women after brain death. Critical care Med 2003;31:1241-9

56 Bernstein IM, Watson M, Simmons GM, Catalano PM, Davis G, Collins R. Maternal brain death and prolonged fetal survival. Obstet Gynecol 1989;74(Part 2):434-7

57 Lane A, Westbrook A, Grady D, et al. Maternal brain death: medical, ethical and legal issues. Intensive Care Med 2004;30:1484-6

(Accepted 22 September 2009) 